\title{
La drogadicción como aspecto social en los adolescentes
}

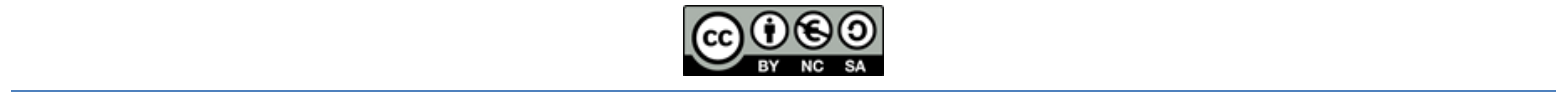

Drug addiction as a social aspect in adolescents

Efraín Velasteguí López. ${ }^{1}$

Recibido: 10-06-2018 / Revisado: 11-06-2018 / Aceptado: 17-06-2018 / Publicado: 06-07-2018

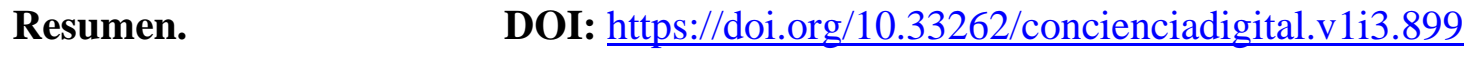

La drogadicción es una enfermedad que hace a la persona depender de sustancias que afectan el sistema nervioso y funciones cerebrales, teniendo diversos efectos que depende del tipo, cantidad o frecuencia que se la consume.

Es considerado un problema social ya que los adolescentes son un grupo muy vulnerable ante las drogas legales e ilegales porque se encuentran con mucha facilidad. Los principales causantes del consumo de drogas son problemas familiares, trastornos psicológicos, emocionales, sociológicos o se encuentran en la etapa de pubertad.

Existen diversos tipos de drogas que cada vez van incrementando su daño debido al proceso para obtener las sustancias, por ejemplo, tenemos la marihuana, crack, estimulantes, etc., como drogas ilegales y como drogas legales el alcohol.

La mayoría de las jóvenes comienzan su consumo debido a la curiosidad o querer aparentar alguien que no son, por problemas familiares, traumas; trayendo consigo consecuencias irreparables como son daños a órganos, vía respiratoria, psicológicos, cerebrales, etc.

La adicción hace aun adolescente más susceptible al robo, asesinatos, ya que al momento de estar drogado la persona se pierde de todos sus sentidos.

El consumo de drogas en la actualidad ha incrementado con gran rapidez por la vulnerabilidad

\footnotetext{
${ }^{1}$ Ciencia digital, Ambato, Ecuador, luisefrainvelastegui@cienciadigital.org
} 
de las autoridades ante el narcotráfico, debido al engaño que sufren las más afectadas son las mujeres porque ellas son utilizadas con mulas para que se lleve a cabo la actividad; también es por personas irresponsables que facilitan a los adolescentes adquirir las drogas legales.

La drogadicción es considerada una plaga que está apoderándose de la mayoría de los pobladores.

Palabras claves: Droga Adolescentes, Estado, Narcotráfico, Plaga, Dependencia, Sobredosis.

\section{Abstract.}

Drug addiction is a disease that makes the person depend on substances that affect the nervous system and brain functions, having various effects that depends on the type, quantity or frequency that is consumed.

It is considered a social problem since adolescents are a group very vulnerable to legal and illegal drugs because they meet very easily. The main causes of drug use are family problems, psychological, emotional, sociological or are in the stage of puberty.

There are different types of drugs that are increasing their damage due to the process to obtain substances, for example we have marijuana, crack, stimulants, etc., as illegal drugs and as legal drugs alcohol.

The majority of young people begin their consumption due to curiosity or wanting to pretend that they are not, due to family problems, traumas; bringing with it irreparable consequences such as damage to organs, respiratory, psychological, cerebral, etc.

The addiction makes an adolescent more susceptible to theft, murders, since at the moment of being drugged the person loses all their senses.

Drug use nowadays has increased very rapidly due to the vulnerability of the authorities to drug trafficking, due to the deception suffered by the most affected women because they are used with mules to carry out the activity; It is also because of irresponsible people who make it easier for teenagers to acquire legal drugs.

Drug addiction is considered a plague that is taking over the majority of people.

Keywords: Drug Teenagers, State, Drug Trafficking, Pest Dependency Overdose. 


\section{Introducción.}

En la época actual no se puede separar el problema de las drogas de los problemas sociales, económicos, políticos y de seguridad que lo acompañan. Esta situación se presenta en cualquier parte del mundo donde se produce, compra, vende, distribuye, exporta, trafica, contrabandea y se consume la droga.(Andres Garrido, 2013).

El problema de la drogadicción en la adolescencia se refiere al abuso de sustancias ilegales o al uso excesivo de las legales. Este patrón de conducta conduce a problemas o preocupaciones graves como: faltar a la escuela, situaciones de peligro, accidentes automovilísticos, problemas legales, con las relaciones familiares y las amistades.(diario, 2011).

También el conocimiento común ha hecho su aporte creando mitos y deformando la realidad, tanto de la drogadicción como el drogadicto formando una discusión al momento de requerir una intervención psicológica encaminada a la rehabilitación o a programas de prevención.(Sanchez, 2006).

\section{Criterio de los autores}

\section{Concepto de drogadicción}

Según (Sanchez, 2006) sostuvo que, "La drogadicción es un estado psíquico y a veces también físico producido por la interacción entre un organismo vivo y un fármaco, caracterizado por comportamientos o reacciones que influye el uso impulsivo de la sustancia".

Llevando como consecuencia al:

Deterioro orgánico

$>$ Deterioro psíquico

$>$ Deterioro social

$>$ Deterioro en la productividad social

\section{Influencia del Estado}

Según (Cardona, Carreño, \& Garcia, 1993) afirman que, "El consumo de drogas es un gran negocio de países, grupos capitalistas del delito y multitud de pequeños delincuentes que sirven como una de las redes de distribución más eficaz del mundo en la interrelación comercial, persona a persona.

Es cierto que hay viejas culturas, especialmente en Asia y América que el consumo de drogas a existido desde siglos como por ejemplo": 
$>$ Marihuana

$>$ Alucinógenos

$>$ Crack

$>$ Estimulantes

Según (H, 2004) opina que, "Las grandes sumas que recibe el Estado en impuestos por comercio y consumo de alcohol y tabaco son insuficientes para cubrir los gastos hospitalarios y carcelarios del país. Más aun, el manejo no cuantificado en el nivel de vida sana, la capacidad de trabajo y estudio general. A esto se suma la alarmante cifra de medio millón de retrasos mentales, como consecuencia del abuso del alcohol y otras sustancias psicoactivas por parte de sus padres.

Se considera que aproximadamente un $20 \%$ de la población latina tiene una o todas las predisposiciones para ser adicto crónico."

\section{Drogas legales e ilegales}

Según (JIFE, 2010)dice que, "En varios países de América del Sur siguió aumentando el abuso de drogas ilícitas, una de las consecuencias del narcotráfico que impera en la subregión. Según la UNODC, cada año casi un millón de personas reciben tratamiento por abuso de drogas ilícitas."

Según (Vega, 2006)opina que, "Las drogas ilegales son parte de nuestra vida cotidiana, prohibirlas es aberrante, más aun cuando el consumo dentro del país es mínimo y su carácter ilegal lo único que hace es impedir que dentro del mercado y/o desde el estado se construyan mecanismos de control que garanticen su calidad y disponibilidad"

\section{Las drogas y los jóvenes}

Según (Hualde, 1998) argumenta que, "Un porcentaje apreciable de jóvenes se drogan habitualmente. Pero un porcentaje mucho mayor no cae en la trampa de la droga, aunque la haya probado ocasionalmente.

Las personas mal estructuradas psíquicamente, desde los inicios de la vida infantil, son presa fácil de la toxicomanía, personas que han sufrido un traumatismo grave en fases anteriores a la adolescencia y no han podido asimilarlo convenientemente.

Muchas más frecuentes son aquellos en los que el adolescente o joven vive un conflicto actual agudo con su entorno familiar, escolar o sentimental.

El "paraíso" de la droga se les aparece como un cómodo refugio que les aporta infelicidad y esclavitud." 


\section{Causas}

Según (Cattaneo, 1999) sostiene que, "Los hijos toman como modelo la identificación a los padres "fantasmas", que nunca están presentes cuando se los necesita o padres "billetera", que suplantan con dinero el compromiso del que carecen. También la inseguridad económica condiciona los proyectos individuales y familiares

El ideal del joven consumidor es una persona o grupo marginado, rechazado por la sociedad; un antihéroe se podría decir, considerado como héroe en lugar de una persona idealizada normalmente, en otros casos se han usado drogas específicamente para comunicarse con poderes sobrenaturales, inalcanzable para ellos por otros medios.

Otros factores que influyen en la drogadicción de un joven son":

$>$ Aburrimiento y ocio

$>$ Anomia

$>$ Súper ego, etc.

Según (Rossi, 2008)argumenta que, "Los padres que no ponen límites actúan de esa manera ya sea porque temen que los hijos los rechacen o porque les resulta más fácil decir siempre que sí. El de rehabilitación con jóvenes que sufren patologías adictivas nos muestra el daño que provocan los padres que no ponen límites. A estos jóvenes adictos no les falta afecto sino patas para aceptar la realidad."

Según (Cañal, 2003)piensa que, "No creo que exista una personalidad adictiva". "Más bien hablaría de factores que pueden ocasionar que un sujeto consuma drogas o no; poco asertividad, escasa tolerancia a la frustración, incapacidad de desarrollar ciertas habilidades. Estas características dependen no solo de la persona, sino también de influencias externas."

Según (Maturana, 2011) sostiene que, "El abuso de nicotina, alcohol y drogas ilegales es una conducta que está siendo cada vez más frecuente en la población adolescente, sobre todo en la asociación a otras conductas de riesgo, tales como violencia, actividad sexual no planificada, etc.

Según (Briones, 2018)argumenta que, "Hay diferentes tipos de drogas, como estimulantes (anfetamina, cocaína, etc.), que afectan los centros nerviosos perjudicándolos en sus movimientos. Depresores (alcohol, barbitúricos, tranquilizantes, morfina, heroína, codeína, inhalantes), que afectan el nivel de atención y provocan depresiones en las personas. Alucinógenos (marihuana, LSD, mezcalina, psilocibina), que como el nombre dice hacen alucinar." 


\section{Tipos de consumidores}

Hay diferentes tipos de consumidores, como:

- EXPERIMENTALES: la persona prueba una vez por curiosidad.

- OCASIONALES: la persona consume una o varias drogas de vez en cuando, sin continuidad, la consume cuando se le presenta la ocasión. No hay búsqueda activa de la sustancia.

- HABITUAL: la persona la consume regularmente, se la ha transformado en un hábito y se preocupa de obtenerla. Son personas que generalmente pueden continuar con sus actividades (estudiar, trabajar,etc.)

- DEPENDIENTE: la persona tiene la necesidad de consumir de forma continuada o periódica la sustancia para evitar los efectos psíquicos o físicos producidos por el síndrome de abstinencia. La obtención y el consumo de la sustancia pasa a la vez a ser la motivación central de su vida.

Según (Rosa, 2003) opina que "El adicto usa como mecanismo las defensas, como la familia. Viven en un sistema basado en la negación y la mentira, la persona se aleja de todas las actividades sociales y familiares, la personalidad de la persona cambia completamente.

Tiene ciertas características tales como:

- La manipulación

- Deshonestidad

- Mentira

El adicto cuando está bajo las influencias de la droga, pierde el control, en ese momento puede matar y también pueden robar.

En la sociedad hay poco interés acerca de la adicción hacia la juventud; la droga ha sido una plaga que continúa matando gente de todas las edades, la droga es fácil de conseguir pero difícil de curar."

Según (Borras, 2017) dice que, "Si pruebas las drogas, te enganchas y te convertirás en un adicto; esto no es cierto. La gran mayoría de los adolescentes y jóvenes que prueban una droga de forma experimental no la vuelve a probar o si lo hacen, la mayoría no abusa de ella." 


\section{Fin de las mujeres dentro del mundo de las drogas}

Según (Torres, 2008) argumenta que "Según los datos analizados anteriormente, decir que el $80 \%$ de las mujeres recluidas son mulas y el hecho de que las mujeres que aceptan hacer este tipo de actividades son mujeres "utilizadas", pues su situación económica permite que se aprovechen de ellas".

\section{Criterio Personal.}

Los adolescentes no entienden el daño que se hacen al momento de consumir las drogas ya sean legales o ilegales, debido a que las dos te dan menos días de vida. Piensan que por hacerlo mucha gente los van a aceptar en su entorno, pero la triste realidad es otra son rechazados y hasta excluidos de la sociedad.

La drogadicción debería ser uno de los temas más importantes en nuestra sociedad porque poco a poco está acabando con el futuro de la patria, pero a nivel mundial las drogas a arrebozadas fronteras y es una plaga casi imposible de terminar.

Las drogas te arrebatan tu libertad ya que dependes de ella al momento de convertirte en un adicto; muchas veces los adictos nacen por problemas familiares, depresión, traumas, y muchos problemas sociales.

Un punto importante es la familia en si los padres debido a que no les dan a entender la diferencia entre la libertad y el libertinaje que la mayoría de los jóvenes no comprenden y piensan que por ser hijos tienen el derecho de muchas cosas.

En fin, ya sea un adolescente o adulto debería buscar otra manera de solucionar sus problemas ya que la droga solamente te da un pequeño momento de relajación pero después de eso todo vuelve hacer lo mismo.

\section{Tabulación y resultados}

Técnica: Encuesta

\begin{tabular}{|c|c|}
\hline Hombre & Mujeres \\
\hline $\mathbf{3}$ & 2 \\
\hline
\end{tabular}

Elaborado por: Fuente Propia 
1.- ¿Qué partes del organismo cree que se ve más afectado por el consumo de drogas?

\section{Gráfico N 1}

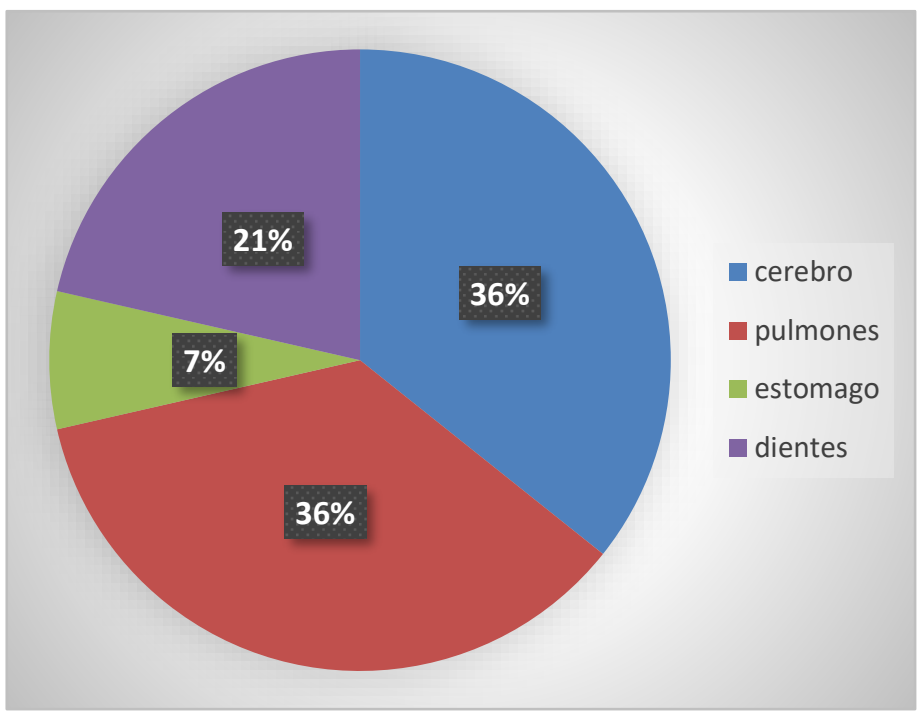

Elaborado por: Fuente Propia

\section{Interpretación}

De 5 personas encuestadas se muestra que 5 de ellas corresponde al 36\% respondió que el cerebro es la parte del organismo más afectada al igual que los pulmones, mientras que 1 de ellas que comprende el $7 \%$ respondió que el estómago, 3 personas que corresponde al $21 \%$ respondió que los dientes.

2.-¿Ha usado drogas alguna vez en su vida?

\section{Gráfico N 2}

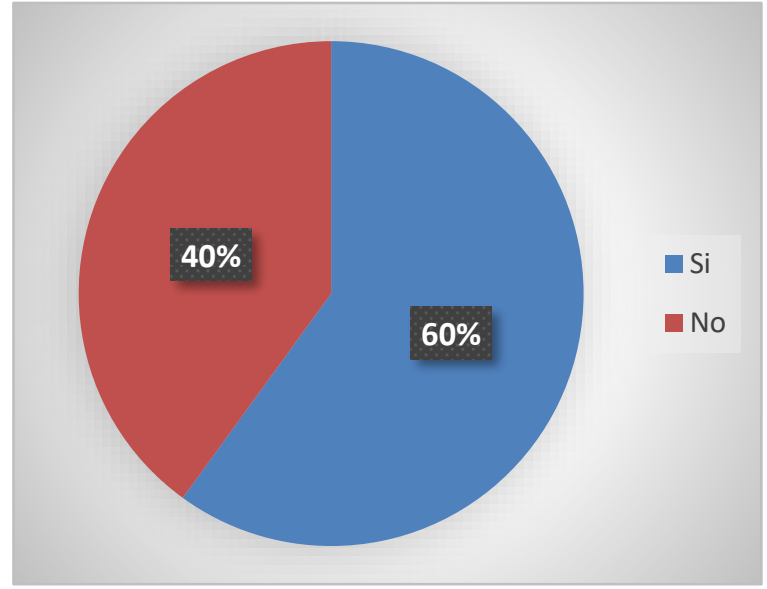

Elaborado por: Fuente Propia 


\section{Interpretación}

De 5 personas encuestadas se muestra que 3 de ellas corresponde al $60 \%$ respondió que $\mathrm{Si}$, mientras que 2 de ellas que comprende el $40 \%$ respondió que No.

3.- ¿Cuál cree que es el motivo más común que lleva a una persona a consumir drogas?

\section{Gráfico N 3}

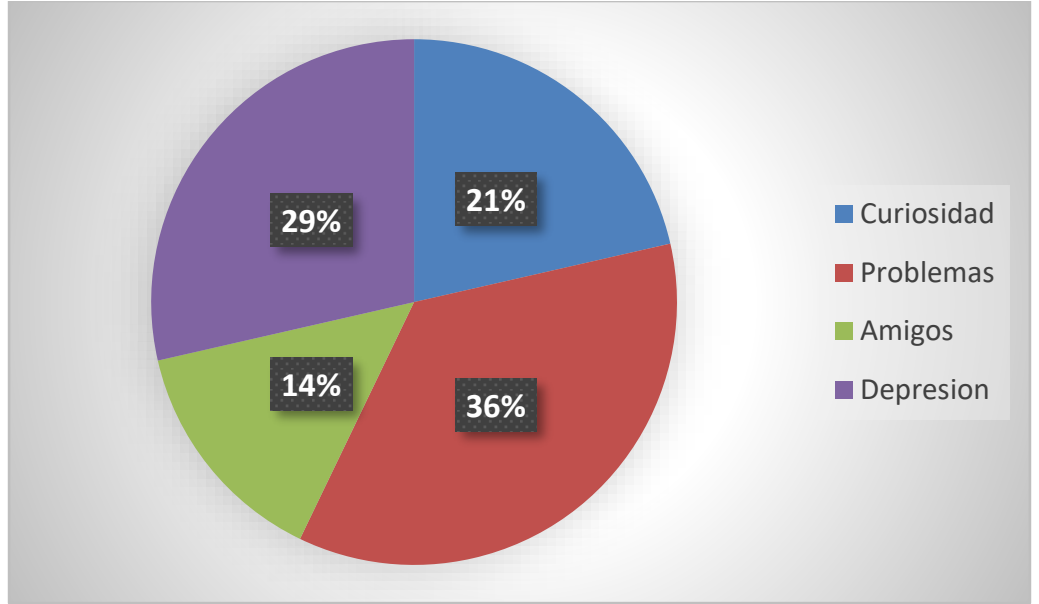

Elaborado por: Fuente Propia

\section{Interpretación}

De 5 personas encuestadas se muestra que 3 de ellas corresponde al $21 \%$ respondió que por curiosidad consumen drogas, mientras que 5 de ellas que comprende el 36\% respondió que debido a problemas, 2 personas que corresponde al 14\% respondió que por los amigos y por ultimo 4 persona que corresponde al $29 \%$ respondió que es por depresión.

\section{4.-¿Qué crees que necesitaran los jóvenes para que dejaran de consumir drogas?}

\section{Gráfico N 4}

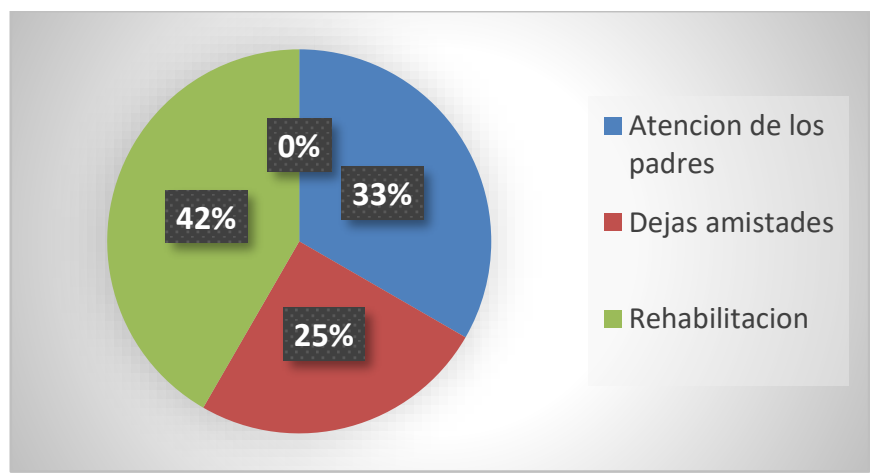

Elaborado por: Fuente Propia 


\section{Interpretación}

De 5 personas encuestadas se muestra que 4 de ellas corresponde al $33 \%$ respondió que necesitarían más atención de los padres, mientras que 3 de ellas que comprende el $25 \%$ respondió que dejar amistades, 5 personas que corresponde al $42 \%$ respondió que la rehabilitación.

\section{5.- ¿Cada cuánto consumes las drogas legales?}

\section{Gráfico N 5}

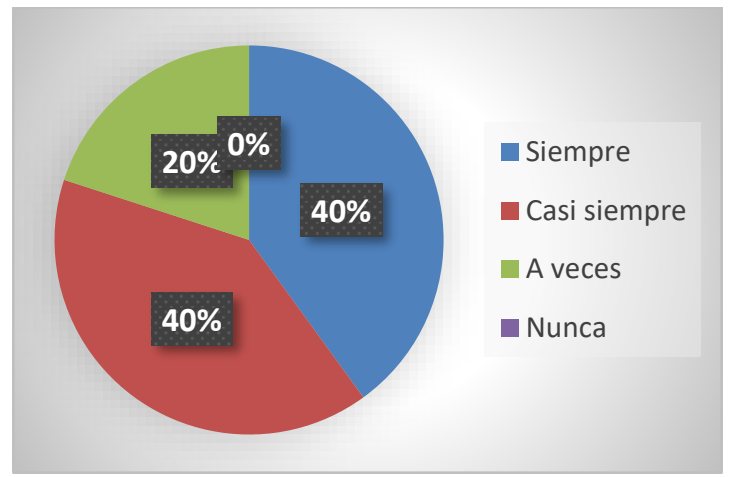

Elaborado por: Fuente Propia

\section{Interpretación}

De 5 personas encuestadas se muestra que 2 de ellas corresponde al $40 \%$ respondió que siempre consumen drogas legales, mientras que 2 de ellas que comprende el $40 \%$ respondió que casi siempre, 1 persona que corresponde al $20 \%$ respondió a veces y ninguna persona respondió nunca.

\section{Conclusiones}

- La drogadicción es un problema que no solo afecta a los adolescentes sino también a los adultos debido a que todos son vulnerables a caer en un vicio pero no todos son capaces de salir de él.

- En la actualidad la droga a rebaza limites debido a que es muy fácil de adquirirla en cualquier parte del mundo, es el dominante de un gran número de población.

- El estado es un influyente en la plaga de la drogadicción porque debido a la corrupción que existe en nuestro país es muy escasa la posibilidad de terminar con el narcotráfico. 
- La mayoría de las jóvenes han probado alguna vez drogas legales e ilegales por curiosidad, pero ello no les hace dependiente de la sustancia, todo depende de la personalidad y autoestima de la persona ya que está en sus manos ser o no ser un adicto más.

\section{Referencias bibliográficas.}

Andres Garrido, E. M. (2013). Cerebro y drogas. guadalajara: Editorial mexicana.

Borras, S. (2017). Guia del uso racional de las drogas. Madrid: copyright.

Briones, W. L. (2018). Causas de las adicciones en adolescentes y jóvenes en Ecuador. Camagüey, vol.22 no.2 .

Cañal, M. (2003). Adicciones . Bogota: Grupo editorial Norma.

Cardona, J., Carreño, P., \& Garcia, E. (1993). No te rindas ante la droga. Madrid: Rialp,S.A.

Cattaneo, R. (1999). Los adolescentes y la problematica de las drogas . Argentina: Juridicas cuyo.

diario, E. n. (1 de Enero de 2011). La drogadicción durante la adolescencia. Obtenido de https://www.elnuevodiario.com.ni/especiales/91436-drogadiccion-duranteadolescencia/

H, J. (2004). Alcoholismo,tabaquismo,drogadiccion soluciones practicas. Colombia : Paulinas.

Hualde, A. (1998). La droga juvenil . Bogota: San pablo.

JIFE. (2010). Informe . Naciones Unidas.

Maturana, A. (2011). COnsumo de alcohol y drogas en adolescentes. Revista Médica Clínica Las Condes, 4-141.

Rosa, M. (2003). Los efectos y las consecuencias de las drogas y el alcohol. Bloomington: congress . 
Rossi, P. (2008). Drogas y los adolescentes. Madrid : tebar .

Sanchez, L. (2006). Mito y realidad de la drogadiccion . Colombia: San pablo.

Torres, A. (2008). Drogas,carcel y genero en Ecuador. Quito: abyayala.

Vega, J. (2006). Caceria de bujos:drogas olegales y sistema de carseles en el ecuador . Quito : abayayala. 
Para citar el artículo indexado.

Velasteguí López, E. (2018). La drogadicción como aspecto social en los adolescentes. ConcienciaDigital, 1(3), 32-44. https://doi.org/10.33262/concienciadigital.v1i3.899

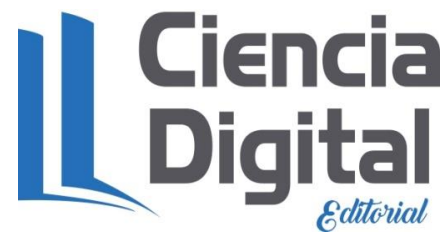

El artículo que se publica es de exclusiva responsabilidad de los autores y no necesariamente reflejan el pensamiento de la Revista Conciencia Digital.

El articulo queda en propiedad de la revista y, por tanto, su publicación parcial y/o total en otro medio tiene que ser autorizado por el director de la Revista Conciencia Digital.
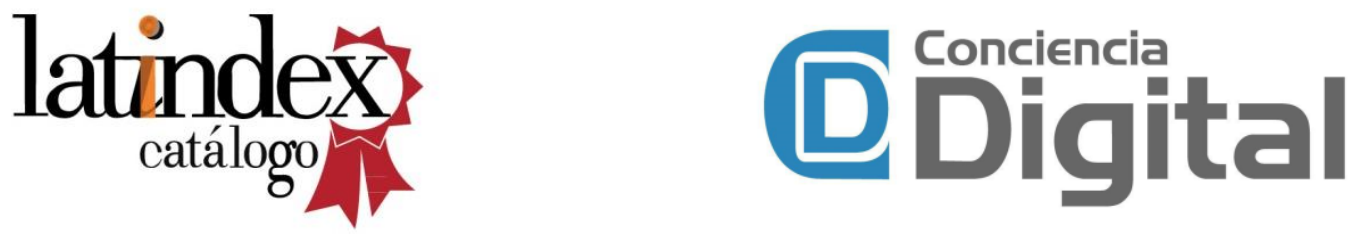